\title{
One year recurrence of trachomatous trichiasis in routinely operated Cuenod Nataf procedure cases in Vietnam
}

\author{
T T K Thanh, R Khandekar, V Q Luong, P Courtright
}

Br J Ophthalmol 2004;88:1114-1118. doi: 10.1136/bjo.2003.039834

Series editors: W V Good and S Ruit

See end of article for authors' affiliations

Correspondence to:

Dr R Khandekar, Eye and Ear Health Care, DGHA, Ministry of Health,

Sultanate of Oman, POB

393, Pin 113, Muscat;

rajshpp@omantel.net.om

Accepted for publication 29 February 2004
Background: Recurrence of trichiasis following surgery remains unacceptably high, regardless of the surgical procedure. Few prospective studies of sufficient size are available to assess the rate of recurrence and the factors contributing to recurrence. A prospective study of the modified Cuenod Nataf surgical procedure was conducted in Vietnam to determine recurrence and co-factors.

Methods: The prospective study of Cuenod Nataf surgery for trachomatous trichiasis took place in four districts of Vietnam. All patients from identified villages who had surgery were followed for a period of 1 year. 10 Surgeons using standard techniques and recording procedures carried out the surgery. The presence of an eyelash touching the eyeball in the operated eye was considered as recurrence. Information on all subjects was recorded preoperatively, intraoperatively, and postoperatively. An independent examiner recorded postoperative information. Relative risks were calculated to assess the contribution of various risk factors to recurrence (by eye and by person). Cox proportional hazards modelling was used to assess the independent contribution of relevant factors to the outcome.

Results: 471 individuals had trichiasis surgery; 463 were followed for a period of 1 year. Overall, the recurrence rate was $10.8 \%$ (95\% Cl 8.0 to 13.6). Among people having surgery recurrence (one or both eyes) was most common in the most elderly (relative risk (RR) 2.49) and among those with a history of previous surgery $(R R=2.49$ ). Cox proportional hazards analysis (by eye) revealed that visual acuity, conjunctival scarring, and suture adjustment were associated with recurrence at 1 year.

Conclusion: The Cuenod Nataf procedure, which is well accepted in the community and by eye care providers in Vietnam, has an acceptable 1 year success rate. Individuals with severe conjunctival scarring have the highest rate of recurrence suggesting that other surgical approaches are needed to manage these patients or that these patients need to be educated regarding the risk of recurrence. Active follow up of these patients would be warranted. The association with suture adjustment requires further investigation.
T imely surgery to prevent vision loss from trachomatous trichiasis is one of the important strategies to achieve the goal of elimination of blinding trachoma by 2020 . Vietnam has set the target of the year 2010 for the elimination of blinding trachoma. ${ }^{1}$ After a well conducted clinical trial of different surgical procedures (performed by ophthalmologists) for trachomatous trichiasis (in Oman) the World Health Organization adopted and promoted bilamellar tarsal rotation (BTR) as the surgical procedure of choice in 1991. ${ }^{2}$ Retrospective and non-concurrent prospective studies carried out since then have demonstrated variable rates of recurrence using this and other procedures. ${ }^{3-8}$ In most cases, the low rate of recurrence detected in the original Oman study has not been replicated elsewhere and adoption of the bilamellar tarsal rotation procedure has not been widespread.

The lowest rate of recurrence $(5 \%$ after 1 year) was reported from Burma ${ }^{8}$ using a procedure generally referred to as the grey line split. ${ }^{8}$ Using the bilamellar tarsal rotation procedure, Ethiopian research detected a recurrence rate (at 3 months postoperation) of $14 \%$. ${ }^{9}$ Another study, using a transverse tarsotomy and lid margin rotation procedure, had a failure rate (at 3 months) of $5 \%$ (for the minor trichiasis cases) and $16 \%$ (for the major trichiasis cases). ${ }^{10}$ In Morocco a modified bilamellar tarsal rotation procedure (generally referred to as the "Trabut procedure") led to a l year recurrence rate $15.8 \%{ }^{1{ }^{11}}$ Finally, a longitudinal study in the Gambia showed a 28\% recurrence rate 1 year after lid surgery. ${ }^{12}$

In early 1990s, ophthalmologists in Vietnam modified and adopted the Cuenod Nataf surgery procedure for correcting trachomatous trichiasis. ${ }^{7}$
Ophthalmologists in Vietnam have been hesitant to adopt the bilamellar tarsal rotation procedure because of previously encountered high failure rates and anticipated poor acceptance by the population. We sought to prospectively evaluate the outcome of the currently used procedure to assist with decision making regarding any change in training, monitoring of outcome, or adoption of a different procedure. We also sought to assess factors, which predict recurrence in our population. We present the outcome of surgery at the end of 1 year.

\section{METHODS}

This was a prospective, community based, outcome study. All enrollees were assessed before surgery, at the time of surgery, l week after surgery, and 1 year after surgery. The study was done including all surgeons who were responsible for trichiasis surgery in the selected districts and provinces.

The provinces of Thanh Hoa, Vinh Phuc, and Ha Nam were selected for the study as they represent areas of relatively low blinding trachoma (Thanh Hoa), moderate blinding trachoma (Vinh Phuc), and high blinding trachoma (Ha Nam) in Vietnam. There are an estimated 16000 trichiasis cases in the three provinces. Within Vinh Phuc and Ha Nam province one district was selected while in Thanh Hoa two districts were selected for inclusion in the study.

A priori, we estimated the 2 year recurrence rate following surgery to be $20 \%$. For a $95 \%$ confidence interval with this rate we required a sample of 242 trichiasis cases. In order to evaluate the potential contributors to recurrence we doubled this number. Communes were listed within each of the identified districts and six, nine, eight, and six communes 
were randomly selected (population proportional to sample) from Binh Loc (Ha Nam province), Tam Duong (Vinh Phuc province), Vinh Loc (Thanh Hoa province), and Tho Xuan (Thanh Hoa province) districts respectively. In each of the selected communes, one village was randomly selected from the list of villages served by the commune. The village health workers in the selected villages (after training) examined all residents over 35 years of age for the presence of trichiasis. All trichiasis patients were referred (and assisted) to the nearby commune health centre for surgery by routinely visiting ophthalmologists. This comprised our study population.

Ten provincial ophthalmologists were the surgeons for the study. All participating ophthalmologists had at least 5 years' experience in the Cuenod Nataf lid surgery procedure. Surgery was carried out in a converted room at the commune health centres. The surgical procedure was further standardised by the supervising surgeon (VQL), who also provided follow up, as required. Standard surgical sets were given to each operating unit. A standard recording form was tested and adopted before the start of the project.

An incision is made at the grey line of the lid margin of the upper lid. The muscle and fibrous tissues are separated from the front surface of tarsus plate by blunt dissection till the upper and lateral edges of the plate are reached. The tarsal plate is fractured horizontally leaving a $3 \mathrm{~mm}$ wide distal piece. In a conventional procedure, a triangular piece of tarsal plate is removed at both the lateral cut ends of the tarsal plate. However, in the modification adopted in Vietnam, this procedure is not carried out. Three equidistant mattress sutures are placed along the width of the lid. These sutures pass from the lid margin, through the cut edge of the distal piece into the anterior surface of the proximal piece of the tarsal plate near the cut edge, then back through the anterior surface of the proximal piece emerging at the cut edge and finally through the cut edge of the distal piece, emerging at the lid margin. During the entire procedure, haemostasis is maintained. The ends of the sutures are tied with one overhand throw, left long, and taped to the brow. Antibiotic ointment is applied to the upper lid and the wound and eye are lightly patched. After 24 hours, the correction is noted. If TT correction is excessive, the knots are loosened and if it is less than desired, the knots are pulled tighter. The sutures are removed on the seventh day. In absence of infection or severe inflammatory response, no systemic antibiotic or antiinflammatory medication is given.

Before surgery the participating ophthalmologists noted demographic and clinical details of the surgical case (age, sex, visual acuity, conjunctival scarring (Dawson), history of epilation, site of the trichiasis (medial, central, or lateral), lid notching, and history of previous lid surgery. All surgeons had a colour copy of the WHO conjunctival scarring grading scheme for comparison and grading of their cases. At the time of surgery, the surgeon recorded the number of sutures (skin and tarsal), fibrosis of the upper fornix, and complications. At the 1 week follow up, information on presence of haematoma and wound gaping were noted. Skin sutures were removed at this time. Need for adjustment of tarsal sutures within 48 hours of surgery was noted. Adjustment of tarsal sutures was indicated if there was undercorrection or overcorrection (as determined by the ophthalmologist). Standard data collection forms were used to report all information. All surgery was provided free of charge.

All surgical cases were targeted for examination at 1 year. These cases were re-examined by four independent field investigators (district ophthalmologists, especially trained for the project) through house to house visits; absentees were revisited. Ophthalmic loupes $(2.5 \times)$ and torch were used during follow up examination to note presence of trichiasis, defined as any eyelash touching the eyeball. We defined "recurrence" as trichiasis in an eye that underwent surgery in the previous year. At the 1 year follow up, vision was also recorded. Those with trichiasis were treated by epilation for temporary relief and then were operated subsequently free of cost.

For all study personnel, standardisation workshops were held to explain the various examination and data collection methodologies. The study methods were pretested in a village outside the study area. The field activities were supervised by one of us (VCL). The data forms were collected and brought to Hanoi for data entry using Epi-6. Data were double checked for consistency. Range and frequency checks were used for validation. Unique identification codes were given to each individual for merging the surgery and follow up data files. The univariate and multivariate analysis was carried out using Statistical Package for Social Studies (SPSS 10). Relative risks (95\% confidence intervals) and p values were used for statistical comparisons. For the purpose of this study, recurrence was defined both by person and by eye. Recurrence assessed by patient undergoing surgery (bilateral or unilateral surgery) was used to assess individual characteristics (for example, age, sex, residence) in order to provide information for eye care providers on the burden in the population and to assist them in counselling patients. Recurrence in individual eyes was analysed to include additional clinical factors that may predict outcome.

Multivariate analysis included use of Cox proportional hazards modelling to assess the independent contribution of demographic and clinical characteristics to recurrence (by person and by eye).

\section{RESULTS}

In the study villages 549 individuals above 35 years of age were identified with trichiasis; all of these individuals underwent interview. Among these 472 accepted surgery $(86 \%)$ and were included in the study; 463 (98.1\%) of them were re-examined 1 year after lid surgery. Two had died and seven were absent. A total of 636 eyes underwent surgery. Bilateral surgery was done on 181 individuals and unilateral surgery was done on 279.

Among the 463 operated individuals 65 had trichiasis on examination at 1 year, 50 in the operated eye, and 15 in an unoperated eye (new case of trichiasis). Among individuals who had surgery $10.8 \%$ (95\% CI 8.0 to 13.6 ) had recurrence in one or both eyes (table 1)

Variables associated with recurrence included elderly age (relative risk 2.49, 95\% CI 1.43 to 4.33), residence in Ha Nam province (relative risk $1.88,95 \%$ CI 1.10 to 3.19 ), and history of previous surgery (relative risk $2.49,95 \%$ CI 1.48 to 4.16 ). Including these variables in a Cox conditional regression model showed that elderly age $(\beta=0.735, \mathrm{SE}=0.309$, $p=0.017)$ and history of previous surgery $(\beta=0.693$, $\mathrm{SE}=0.293, \mathrm{p}=0.018$ ), but not residence in Ha Nam province remained associated with recurrence. The clinical disease varied between the provinces; for example, Ha Nam surgical cases were significantly older (59.6\% age 70 and above) than Thanh Hoa cases (42.9\% age 70 and above) and Vinh Phuc cases (28.4\% age 70 and above). Severe conjunctival scarring was not as common in Thanh Hoa $(7.5 \%)$ as in Vinh Phuc (36.3\%) or Ha Nam (34.8\%).

Among the operated eyes, recurrence of trichiasis (table 2) was associated with visual acuity, severe conjunctival scarring (relative risk $7.86,95 \%$ CI 2.87 to 21.50 ), as well as elderly age (relative risk $2.48,95 \%$ CI 1.47 to 4.17 ), history of previous surgery (relative risk $2.32,95 \%$ CI 1.42 to 3.80 ), and adjustments made to sutures (relative risk 2.31, 95\% CI 1.37 to 3.92). Ophthalmologists adjusted sutures on the second postoperative day in 110 eyes; among them recurrence was 


\begin{tabular}{|c|c|c|c|}
\hline & $\begin{array}{l}\text { No recurrence } \\
\text { No (\%) }\end{array}$ & $\begin{array}{l}\text { Recurrence } \\
\text { No (\%) }\end{array}$ & $\begin{array}{l}\text { RR }(95 \% \mathrm{Cl}) \\
\text { p value }\end{array}$ \\
\hline \multicolumn{4}{|l|}{ Sex } \\
\hline Males & $102(91.9)$ & $10(8.9)$ & \\
\hline Females & 311 (88.6) & 40 (11.4) & $1.28(0.66$ to 2.47$)$ \\
\hline \multicolumn{4}{|l|}{ Age group } \\
\hline$<50$ years & $40(100)$ & 0 & \\
\hline $50-59$ & 79 (92.9) & $6(7.1)$ & \\
\hline $60-69$ & $124(91.9)$ & $11(8.1)$ & \\
\hline $70+$ & 170 (83.7) & 33 (16.3) & $\begin{array}{l}2.49(1.43 \text { to } 4.33) \\
p<0.001\end{array}$ \\
\hline \multicolumn{4}{|l|}{ Province } \\
\hline $\mathrm{Ha} \mathrm{Nam}$ & 95 (83.3) & 19 (16.7) & $1.88(1.10$ to 3.19$)$ \\
\hline Vinh Phuc & $94(92.2)$ & $8(7.8)$ & $p=0.03$ \\
\hline $\begin{array}{l}\text { Thanh Hoa } \\
\text { Surgeon }\end{array}$ & \multicolumn{3}{|c|}{ Surgeon } \\
\hline A & $65(90.3)$ & $7(9.7)$ & $\chi^{2}=12.97$ \\
\hline B & $86(95.6)$ & $4(4.4)$ & $\tilde{d f}=7$ \\
\hline C & $36(90.0)$ & $4(10.0)$ & $p=0.07$ \\
\hline D & $32(82.1)$ & 7 (17.9) & \\
\hline $\mathrm{E}$ & $57(91.9)$ & $5(8.1)$ & \\
\hline $\mathrm{F}$ & $14(92.3)$ & $1(6.7)$ & \\
\hline G & $52(80.0)$ & $13(20.0)$ & \\
\hline $\mathrm{H}$ & $46(88.5)$ & $6(11.5)$ & \\
\hline 1 & $26(92.9)$ & $2(7.1)$ & \\
\hline \multicolumn{4}{|c|}{ History of lid surgery } \\
\hline No & $316(92.4)$ & $26(7.6)$ & \\
\hline Yes & $103(81.1)$ & $24(18.9)$ & $\begin{array}{l}2.49(1.48 \text { to } 4.16) \\
p<0.001\end{array}$ \\
\hline \multicolumn{4}{|l|}{ Surgery on } \\
\hline One eye & $253(91.7)$ & $23(8.3)$ & \\
\hline Both eyes & $155(86.1)$ & 25 (13.1) & 1.77 (0.93 to 3.37$)$ \\
\hline
\end{tabular}

recognised in 18 eyes (16.4\%). Recurrence was recognised in $36(7.1 \%)$ of 509 eyes in which sutures were not adjusted.

Cox conditional regression modelling demonstrated that conjunctival scarring (hazards ratio 4.59 , 95\% CI 3.40 to 6.19), blindness (hazards ratio $2.20,95 \%$ CI 1.65 to 2.94 ), and suture adjustment (hazards ratio $1.13,95 \%$ CI 1.06 to 1.20 ) were independently associated with recurrence at 1 year. Neither age nor history of previous surgery remained associated with recurrence.

The incidence of new trichiasis in patients who did not have trichiasis at baseline was 15/267 (5.6\%, 95\% CI 2.9 to 8.4). New trichiasis was associated with residence in Ha Nam province (all 15 cases), female sex (11 cases), elderly age group ( 14 cases), and severe conjunctival scarring ( 10 cases).

\section{DISCUSSION}

The epidemiological pattern of trachoma in Vietnam, similar to most trachoma endemic countries, varies considerably owing to the focal nature of the disease. It is likely that the variable pattern of disease will also contribute to variable recurrence rates following surgery. Previous research in Oman has shown that recurrence can be higher in some districts versus others, regardless of the surgeon. ${ }^{4}$ Trichiasis patients who live in Ha Nam province, known to have a long term history of trachoma as a severe public health problem, have more severe conjunctival scarring and are older; in our study they are more likely to have recurrent trichiasis. Furthermore, Ha Nam patients without trichiasis at baseline were more likely to develop incident trichiasis. While the surgical skills of the participating ophthalmologists cannot be completely ruled out as contributing to the recurrence rate, it should be noted that there were no cases of surgical failure at l week postoperation. Province of residence (and, by default, surgeon) were no longer associated with recurrence once logistic regression modelling was used to control for the presence of conjunctival scarring, age, and other factors associated with outcome.

Elderly age, found to be predictive of recurrence in other settings, ${ }^{4}$ was also predictive of recurrence in the Vietnamese subjects. The elderly have the highest prevalence of severe conjunctival scarring, and most of the excess recurrence of trichiasis in the elderly can be attributed to the high prevalence of severe conjunctival scarring in this group. It is likely that severe scarring leads to additional contracture of the tarsal tissues leading to trichiasis. It is interesting to note that the incidence of new trichiasis (in eyes without trichiasis at baseline) was $0 \%$ ( $0 / 82$ eyes ), 3.4\% (5/149 eyes), and 28.6\% (10/35 eyes) for eyes with mild, moderate, and severe baseline conjunctival scarring, respectively. Nevertheless, the contribution of elderly age remained predictive of recurrence after controlling for conjunctival scarring but the possible reasons for this association remain unknown.

Unlike recent research on tarsal rotation surgery from Ethiopia, ${ }^{13}$ preoperative location of the lashes (central versus medial or lateral) did not predict recurrence in the Vietnamese study population. This may simply be due to the different surgical procedure, which had uniform correction at the central and lateral part of lids. It could also be to because of the difference in lid structure of oriental and African populations.

It was not the purpose of this study to compare the modified Cuenod Nataf procedure with the bilamellar tarsal rotation procedure and it is not possible to judge if the clinical outcomes we have recorded could have been better or worse using the bilamellar tarsal rotation procedure. Furthermore, it was not the aim of this study to measure the incidence of new trichiasis in eyes without trichiasis at baseline. The study population is considered "high risk" as there is trichiasis in one eye already; these patients are probably more likely to develop trichiasis in the other eye compared to age matched individuals without trichiasis in either eye. Nevertheless, the 
Table 2 Clinical characteristics of eyes that had recurrence of trichiasis at 1 year postoperation

\begin{tabular}{|c|c|c|c|}
\hline Parameters & $\begin{array}{l}\text { No recurrence } \\
\text { No (\%) }\end{array}$ & $\begin{array}{l}\text { Recurrence } \\
\text { No (\%) }\end{array}$ & $\begin{array}{l}\text { RR }(95 \% \mathrm{Cl}) \\
\text { p value }\end{array}$ \\
\hline \multicolumn{4}{|l|}{ Demographic } \\
\hline Males & \multicolumn{3}{|c|}{ Sex } \\
\hline Females & $431(90.4)$ & $46(9.6)$ & 1.39 (0.74 to 2.62 ) \\
\hline \multicolumn{4}{|l|}{ Age group } \\
\hline$<50$ years & $58(100)$ & 0 & \\
\hline $50-59$ & $112(94.9)$ & $6(5.1)$ & \\
\hline $60-69$ & $174(92.6)$ & $14(7.4)$ & \\
\hline $70+$ & $235(85.4)$ & $37(13.6)$ & $\begin{array}{l}2.48(1.47 \text { to } 4.17) \\
p<0.001\end{array}$ \\
\hline \multicolumn{4}{|l|}{ Province } \\
\hline Vinh Phuc & $147(93.6)$ & $10(6.4)$ & 1.0 \\
\hline $\mathrm{Ha} \mathrm{Nam}$ & $153(89.0)$ & $19(11.0)$ & 1.73 (0.83 to 3.62$)$ \\
\hline Thanh Hoa & $279(90.9)$ & $28(9.1)$ & $1.43(0.71$ to 2.87$)$ \\
\hline \multicolumn{4}{|l|}{ Surgeon } \\
\hline A & $84(92.3)$ & $7(7.7)$ & $\chi^{2}=12.02$ \\
\hline B & $92(95.8)$ & $4(4.2)$ & $d f=7$ \\
\hline C & $52(89.7)$ & $6(10.3)$ & $p=0.15$ \\
\hline D & $46(82.1)$ & $10(17.9)$ & \\
\hline $\mathrm{E}$ & 83 (93.3) & $6(6.7)$ & \\
\hline $\mathrm{F}$ & $21(95.5)$ & $1(4.5)$ & \\
\hline G & $90(87.4)$ & $13(12.6)$ & \\
\hline $\mathrm{H}$ & $68(91.9)$ & $6(8.1)$ & \\
\hline I & $47(94.0)$ & $3(6.0)$ & \\
\hline \multicolumn{4}{|l|}{ Clinical } \\
\hline \multicolumn{4}{|l|}{ Visual acuity } \\
\hline Less than $6 / 60$ & $91(77.8)$ & $26(22.2)$ & $\begin{array}{l}3.67(2.27 \text { to } 5.94) \\
p<0.001\end{array}$ \\
\hline $6 / 60$ to $6 / 18$ & $384(92.5)$ & $31(7.5)$ & \\
\hline Better than $6 / 18$ & $97(100)$ & 0 & \\
\hline \multirow{2}{*}{\multicolumn{4}{|c|}{ Conjunctival scarring }} \\
\hline & & & \\
\hline Mild & $127(96.9)$ & $4(3.1)$ & 1.0 \\
\hline Moderate & $327(95.1)$ & $17(4.9)$ & 1.62 (0.55 to 4.72 ) \\
\hline Severe & $114(76.0)$ & $36(24.0)$ & $\begin{array}{l}7.86(2.87 \text { to } 21.5) \\
p<0.001\end{array}$ \\
\hline \multicolumn{4}{|c|}{ History of previous surgery } \\
\hline No & $453(93.4)$ & $32(6.6)$ & \\
\hline Yes & $138(84.7)$ & $25(15.3)$ & $\begin{array}{l}2.32(1.42 \text { to } 3.80) \\
p<0.001\end{array}$ \\
\hline \multicolumn{4}{|l|}{ Site of trichiasis } \\
\hline Medial third & $360(89.9)$ & $41(10.2)$ & 1.65 (0.89 to 3.07 ) \\
\hline Central & $118(95.9)$ & $5(4.1)$ & \\
\hline Lateral third & $64(90.1)$ & $7(9.9)$ & \\
\hline \multicolumn{4}{|c|}{ Suture adjustments made } \\
\hline No & $473(92.9)$ & $36(7.1)$ & \\
\hline \multirow{2}{*}{\multicolumn{4}{|c|}{ Lid notching }} \\
\hline & & & \\
\hline No & $574(91.3)$ & $55(8.7)$ & \\
\hline Yes & $13(92.9)$ & $1(7.1)$ & 0.82 (0.12 to 5.49 ) \\
\hline \multicolumn{4}{|l|}{ Dysplastic lashes } \\
\hline No & $572(91.7)$ & $52(8.3)$ & \\
\hline Yes & $19(79.2)$ & $5(20.8)$ & 2.50 (1.10 to 5.69$)$ \\
\hline \multicolumn{4}{|c|}{ Fibrosis of upper fornix } \\
\hline No & $452(93.9)$ & $36(6.1)$ & \\
\hline Yes & $138(86.8)$ & $21(13.2)$ & $\begin{array}{l}1.79 \text { (1.08 to } 2.97) \\
\text { p0.03 }\end{array}$ \\
\hline \multicolumn{4}{|c|}{ Number of tarsal sutures } \\
\hline$\leqslant 3$ & $125(91.2)$ & $12(8.8)$ & $\chi^{2}=0.65, d f=2, p=0.72$ \\
\hline 4 & $165(92.7)$ & $13(7.3)$ & \\
\hline$\geqslant 5$ & $268(90.5)$ & $28(9.5)$ & \\
\hline Number of skin s & & & \\
\hline 0 & $158(94.0)$ & $10(6.0)$ & $\chi^{2}=11.8, d f=4, p=0.02$ \\
\hline 3 & $93(95.9)$ & $4(4.1)$ & \\
\hline 4 & $83(83.8)$ & $16(16.2)$ & \\
\hline 5 & 77 (90.6) & $8(9.4)$ & \\
\hline 6 & $115(88.5)$ & $15(11.5)$ & \\
\hline Complications & & & \\
\hline No & $573(91.1)$ & $56(8.9)$ & \\
\hline Yes & $16(94.1)$ & $1(5.9)$ & 0.66 (0.10 to 4.50$)$ \\
\hline
\end{tabular}


findings suggest that trichiasis surgical services will be required for many years to come in Vietnam, particularly in Ha Nam. Either periodic active screening or passive promotion of surgical services is needed to ensure that these patients receive surgery.

Readjustment of the suture (in order to overcorrect/ undercorrect, according to clinical assessment) rather than being beneficial was predictive of recurrence. This finding may be because readjustment of the suture could be permitting the distant segment of the tarsal plate to roll back to its original position resulting in recurrence. Further investigation of this practice and its consequences is needed. It should be noted that adjustments of suture postoperatively were associated with recurrence but not necessarily the cause of recurrence.

This is the first report of follow up in people undergoing the modified Cuenod Nataf procedure. Overall, our findings suggest that the short term recurrence rate of the modified Cuenod Nataf procedure in Vietnam is acceptable. This procedure is well accepted by the community, can be done in commune health centres, and is commonly taught and practised throughout Vietnam. Careful counselling of patients is needed so those with severe conjunctival scarring and those with blindness are aware that recurrence is possible. Long term follow up information is needed to determine if, over time, the recurrence rate rises and, if so, what factors contribute to the increase in recurrence.

\section{ACKNOWLEDGEMENTS}

The authors acknowledge the support extended and permission granted for the study by the Ministry of Health at national, provincial, and district levels. The financial and technical support of International Trachoma Initiatives and the British Columbia Center for Epidemiologic and International Ophthalmology, Canada, is much appreciated. We are also grateful for the assistance provided by Mr Stephan Lagree, Dr Susan Lewallen, and Dr Silvio Mariotti.
The community and patient participation of this community based research was unique and we thank them.

\section{Authors' affiliations}

T T K Thanh, V Q Luong, National Institute of Ophthalmology, Hanoi, Vietnam

R Khandekar, P Courtright, British Columbia Centre for Epidemiologic and International Ophthalmology, Vancouver, Canada

P Courtright, Kilimanjaro Centre for Community Ophthalmology, Moshi, Tanzania

\section{REFERENCES}

1 Thanh TTK. Report of the fourth meeting of the WHO Alliance for the Global Elimination of Blinding Trachoma December 1999. Geneva: World Health Organization, WHO/PBD/00, 9, pp 19-21.

2 Reacher MH, Foster A, Huber A. Trichiasis surgery for trachoma. The bilateral tarsal rotation procedure. Geneva: World Health Organization, WHO/PBL/ $93,29,1991$. 3 Reacher MH, Munouz B, Al Ghassany, et al. A controlled trial of surgery for
trachomatous trichiasis of the upper lid. Arch Ophthalmol 1992;1 10:667-74.

4 Khandekar R, Mohammed AJ, Courtright P. Recurrence of trichiasis: a longterm follow-up study in the Sultanate of Oman. Ophthalmic Epidemiol 2001;8:155-61.

5 Yeung YM, Hon CY, Ho CK. A simple surgical treatment for upper lid trichiasis. Ophthalmic Surg Lasers 1997;28:74-6.

6 Bog H, Yorston D, Foster A. Results of community-based eyelid surgery for trichiasis due to trachoma. $\mathrm{Br} J$ Ophthalmol 1993;77:81-3.

7 Cornand G, Doury JC. Trachomatous tarsitis: its consequences and their surgical treatment. Med Trop 1981;41:157-71.

8 Win N. Grey line split surgery in trichiasis. Rev Int Trach Pathol Ocul Trop Subtrop 1976;53:111-28.

9 Zerihun N. Trachoma in Jimma zone, south western Ethiopia. Trop Med Int Health 1997;2:1115-21.

10 Thommy CP. A modified technique for correction of trachomatous cicatricial entropion. Br J Ophthalmol 1980;64:296-8.

11 Negrel AD, Chami-Khazraii Y, Arrache ML, et al. The quality of trichiasis surgery in the kingdom of Morocco. Sante 2000;10:81-92.

12 Bowman RJ, Faal H, Myatt M, et al. Longitudinal study of trachomatous trichiasis in the Gambia. BrJ Ophthalmol 2002;86:339-43.

13 Melese M, Alemayehu W, Bejiga A, et al. Modified grading system for upper eyelid trachomatous trichiasis. Ophthalmic Epidemiol 2003;10:75-80. 